\title{
Breastfeeding restored the gut microbiota in caesarean section infants and lowered the infection risk in early life
}

\author{
Cheng Guo ${ }^{1 \dagger}$, Qian Zhou ${ }^{2 \dagger}$, Muxia $\mathrm{Li}^{3}$, Letian Zhou ${ }^{4}$, Lei Xu ${ }^{5}$, Ying Zhang ${ }^{1}$, Dongfang Li ${ }^{4}$, Ye Wang ${ }^{1}$, Wenkui Dai ${ }^{4}$, \\ Shuaicheng $\mathrm{Li}^{2}$ and Lin Zhang ${ }^{1 *}$
}

\begin{abstract}
Background: The initialization of the neonatal gut microbiota (GM) is affected by diverse factors and is associated with infant development and health outcomes.

Methods: In this study, we collected 207 faecal samples from 41 infants at 6 time points (1, 3, and 7 days and 1, 3, and 6 months after birth). The infants were assigned to four groups according to delivery mode (caesarean section (CS) or vaginal delivery (VD)) and feeding pattern (breastfeeding or formula milk).

Results: The meconium bacterial diversity was slightly higher in CS than in VD. Three GM patterns were identified, including Escherichia/Shigella-Streptococcus-dominated, Bifidobacterium-Escherichia/Shigella-dominated and Bifidobacterium-dominated patterns, and they gradually changed over time. In CS infants, Bifidobacterium was less abundant, and the delay in GM establishment could be partially restored by breastfeeding. The frequency of respiratory tract infection and diarrhoea consequently decreased.
\end{abstract}

Conclusion: This study fills some gaps in the understanding of the restoration of the GM in CS towards that in VD.

Keywords: Gut microbiota, Early life, Meconium, Delivery mode, Feed pattern

\section{Background}

The intestinal tract hosts millions of microbial colonizers, and the gut microbiota $(\mathrm{GM})$ is positively associated with human health $[1,2]$. A wide variety of reports have demonstrated that caesarean section (CS) blocks gut and vaginal microbiota transmission from mothers to neonates, which delays subsequent health development $[2,3]$. The feeding types also significantly shape the composition of the GM in infancy [4]. As human milk contains a high proportion of probiotics, prebiotics and active molecules, $[5,6]$

\footnotetext{
*Correspondence: Izhang_79@163.com

${ }^{+}$Cheng Guo and Qian Zhou contributed equally to this work.

'Department of Pediatrics, The Third Hospital of Hebei Medical University, No.139 Ziqiang Road, Shijiazhuang 050051, China

Full list of author information is available at the end of the article
}

breastfeeding is more beneficial to GM maturation and health than formula feeding $[7,8]$.

Recent analyses have revealed that human milk promoted the functional maturation of GM after parturition [8]. A series of studies indicated that GM maturation was positively associated with pediatric health in the early life, named the window of opportunity [9-12]. Moreover, the delayed establishment of GM impacted infant development and increased the risks of disease pathogenesis during development $[8,13]$. Considering the differences between Chinese and Western populations, such as differences in environment and diet, we collected 207 faecal samples from 41 Chinese neonates at six time points (in $24 \mathrm{~h}$ after birth, 48-72 $\mathrm{h}$ after delivery, and 7 days, 1,3 , and 6 months of age). We aimed to reveal whether breastfeeding could restore the GM established in CS towards

(c) The Author(s). 2020 Open Access This article is licensed under a Creative Commons Attribution 4.0 International License, which permits use, sharing, adaptation, distribution and reproduction in any medium or format, as long as you give appropriate credit to the original author(s) and the source, provide a link to the Creative Commons licence, and indicate if changes were made. The images or other third party material in this article are included in the article's Creative Commons licence, unless indicated otherwise in a credit line to the material. If material is not included in the article's Creative Commons licence and your intended use is not permitted by statutory regulation or exceeds the permitted use, you will need to obtain permission directly from the copyright holder. To view a copy of this licence, visit http://creativecommons.org/licenses/by/4.0/ The Creative Commons Public Domain Dedication waiver (http://creativecommons.org/publicdomain/zero/1.0/) applies to the data made available in this article, unless otherwise stated in a credit line to the data. 
that in vaginal delivery (VD) and lower the risk of infections in early life $[7,14]$.

\section{Methods \\ Participant enrolment}

The infants were enrolled from the Third Hospital of Hebei Medical University between Dec 2011 and Apr 2013. The inclusion criteria for mothers were as follows: i) no family allergy history; ii) no obesity, diabetes, allergic diseases, cardiovascular diseases or constipation during pregnancy; iii) full-term labour $(>=37$ gestational weeks); iv) infants were fed by pure human milk (B group) or pure formula milk without prebiotics (F group). In combination with the mode of delivery (CS or $\mathrm{VD})$, the enrolled children were assigned into four groups (VD_B, VD_F, CS_B and CS_F).

\section{Sample collection}

During the regular examination at 6 time points $(1,3$, and 7 days and 1, 3, and 6 months after birth), all faecal samples were collected under a nurse's guidance using sample swabs (iClean, Huachenyang (Shenzhen) Technology Co., LTD, China) and stored in sterilized tubes (62-558-201, SARSTEDT AG \& Co., KG, Germany). The collected samples were transferred to a $-80^{\circ} \mathrm{C}$ freezer within 30 min after collection for long-term storage. Respiratory tract infection (RTI) and diarrhoea were recorded during the first year of life (Supplementary File 1). A total of 207 stool samples from 41 infants were collected between December 2011 and October 2014.

\section{DNA preparation and sequencing}

Faecal bacterial DNA was extracted with the E.Z.N.A. DNA Kit (Omega BioTek, Norcross, GA, United States), and then, the V3-4 region of the $16 \mathrm{~S}$ rDNA gene was amplified by the primers 338F (ACTCCTACGGGAGG CAGCAG) and 806R (GGACTACHVGGGTWTCTAAT) using a PCR kit (TransGenAP221-02, Peking, China). The verified amplicon products were then used to construct an amplicon library. Then, high-throughput DNA sequencing was conducted on the MiSeq platform (Illumina, San Diego, CA, United States).

\section{Bioinformatics analysis}

The raw sequencing reads were filtered by Mothur software (v.1.43.0) with our in-house optimized scripts [15, 16]. The raw reads meeting any of the following criteria were removed: i) contained adapter sequences, ii) accumulated low-quality bases (lower than 20) at more than $10 \%$ of the read length. Then, the filtered paired reads were connected to tags with $10 \mathrm{bp}$ overlaps. Tags were then clustered into operational taxonomic units (OTUs) using the Usearch method (v.10.0) [17]. Taxonomical annotation of OTUs was conducted using the RDP classifier (v.2.2) against the Greengenes database (v 13.5). Bacterial diversity was calculated by Mothur software, and the confounding effect of phenotypes was assessed through permutational multivariate analysis of variance (PERMANOVA). The stratification analysis of the delivery mode and feeding patterns was conducted by NMDS. The samples were assigned to the representative clusters based on the relative abundances of different microbial components according to the MetaHIT enterotype calculation method [18].

\section{Statistical analysis}

The chi-square test was applied to analyse categorical variable differences, and one-way analysis of variance was used to assess continuous variables. The Wilcoxon ranksum test was applied to evaluate significant differences in bacterial diversity and abundance between groups. Multiple statistical results from the Wilcoxon rank-sum test were adjusted with the Benjamini and Hochberg method (FDR < 0.05) using "p.adjust" in R (v. 3.6.0).

\section{Results}

All microbial samples were assigned to four groups according to delivery mode (VD and CS) and feeding pattern (breastfeeding, B; formula milk, F): VD_B (14 infants with 69 samples), VD_F (10 infants with 53 samples), CS_B (7 infants with 31 samples) and CS_F (10 infants with 54 samples) (Fig. 1, Table 1). There were no significant differences in infant gender, gestational age or mother's age (Table 1, Supplementary File 1) between groups. Breastfeeding was significantly associated with a lower incidence of RTI and diarrhoea in both VD and CS infants ( $P$-value $<0.001$ and $<0.001$, Table 1$)$. In addition, PERMANOVA showed that the feeding pattern was the most dominant factor shaping the GM in the first 6 months $(P$-value $=0.004)$.

Although insignificant, the GM diversity in CS neonates $(3.18 \pm 0.68)$ was higher than that in VD neonates $(3.01 \pm$ 1.51 ) at six time points (Supplementary File 2A). Compared to CS infants, Bifidobacterium was enriched nearly two-fold in the VD infants' meconium $(20.70 \% \pm 20.01)$ (Supplementary File 3). Other accumulated microbial components in CS infants included Escherichia/Shigella, Enterococcus, Streptococcus, Burkholderia, Acinetobacter, Lactobacillus and Ralstonia (Supplementary File 3).

The 207 faecal samples collected were classified into 3 clusters according to GM structure. Escherichia/Shigella and unclassified taxa dominated the GM in Cluster 1, while Bifidobacterium and unclassified taxa were dominant in the GM of Cluster 2 (Fig. 1a). In Cluster 3, Bifidobacterium was the most abundant genus in the GM (Fig. 1a). In the first week, the Cluster1 GM pattern was identified in most of the samples (Fig. 1b), and the relative abundances of Enterococcus and Escherichia/Shigella 
A

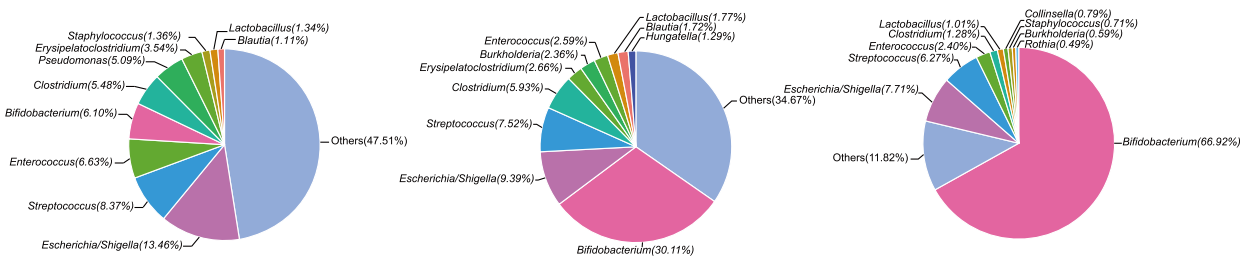

B

Cluster1

Cluster2

Cluster3
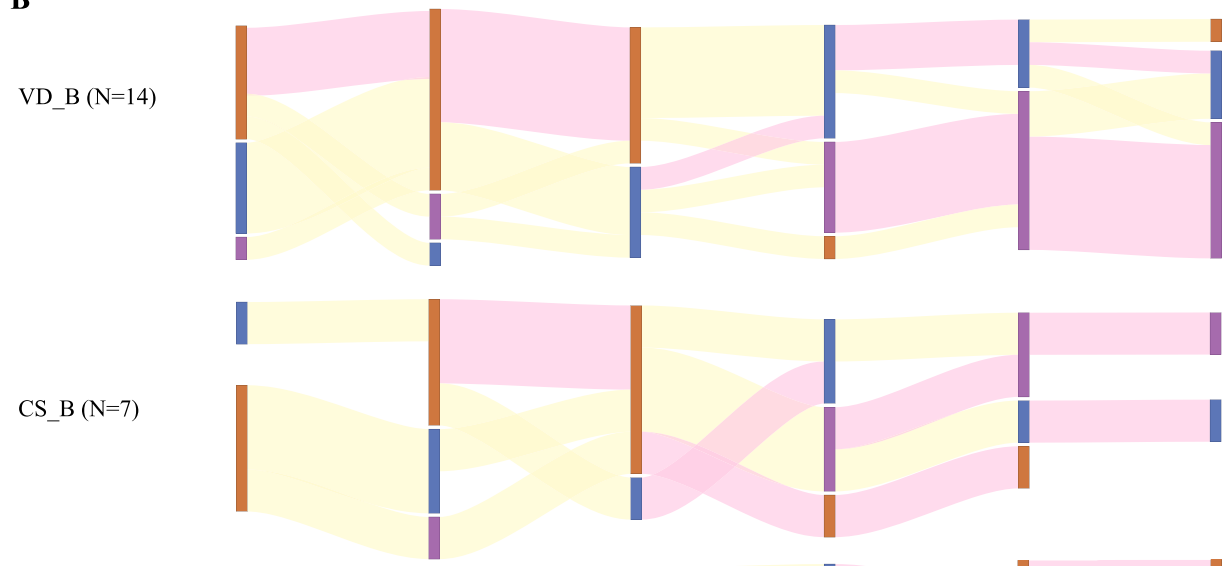

VD_F (N=10)
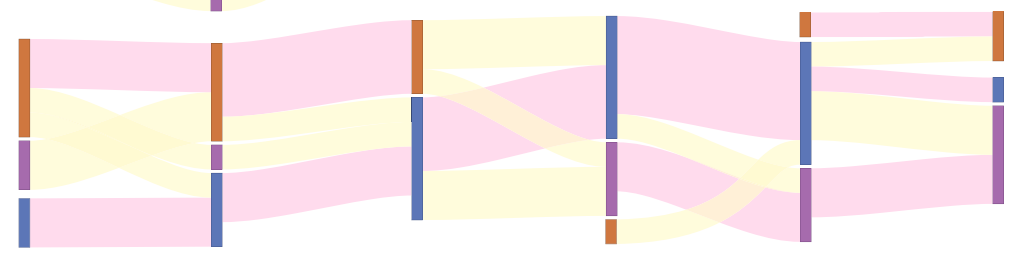

CS_F $(\mathrm{N}=10)$
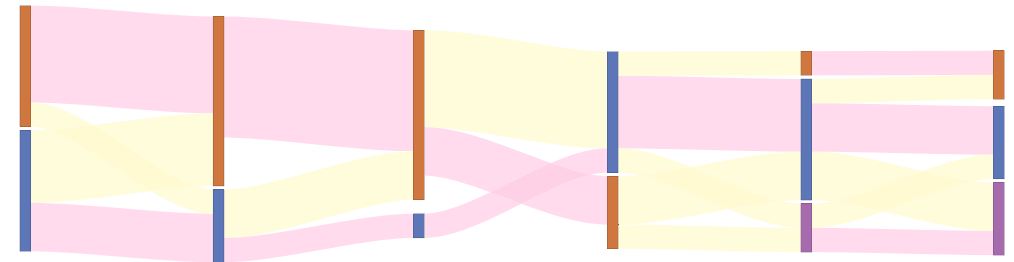

C

D1

D3

D7

Time points

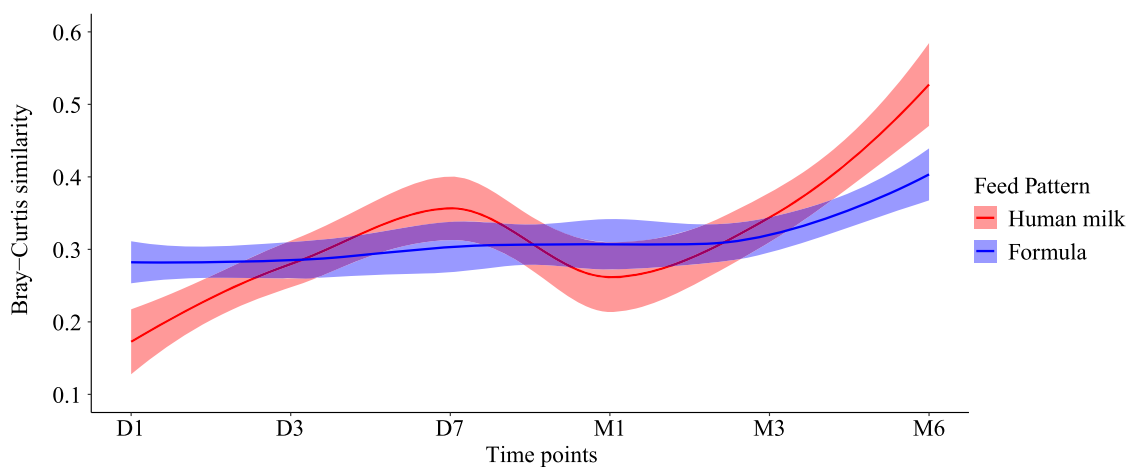

Fig. 1 (See legend on next page.) 
(See figure on previous page.)

Fig. 1 The patterns of the infant gut microbiota changed dynamically over time. a The GM clustered into 3 patterns. Each pie chart represents one GM pattern with the top 10 bacteria and others. GM Cluster 1 is Escherichia/Shigella-Streptococcus dominant and is coloured orange, Cluster 2 is Bifidobacterium-Escherichia/Shigella dominant and is coloured blue, and Cluster 3 is Bifidobacterium dominant and is coloured purple. $\mathbf{b}$ The dynamic change in GM patterns during the first half-year was different in four groups, including VD_B (vaginal delivery and breastfeeding), CS_B (caesarean section and breastfeeding), VD_F (vaginal delivery and formula feeding) and CS_F (caesarean section and formula feeding). The bar colour represents the GM pattern, and the bar length represents the proportion. The ribbon between bars indicates the changes in the GM pattern, where pink represents maintenance and yellow represents change. c Beta-diversity measured the difference in microbiota between CS and VD with age. Human milk (red colour) is better at restoring the infant gut microbiota than formula (blue colour)

increased slightly (Supplementary File 3). The abundance of the meconium-dominant Pseudomonas decreased sharply on day 3 , especially in infants receiving breastfeeding (P-value $=0.004,0.028$ in VD_B and CS_B $)$ (Supplementary File 3). During the neonatal period, especially from D7 to M1, the GM composition shifted from Cluster 1 to Cluster 2 or Cluster 3 (Fig. 1b). Bifidobacterium was significantly enriched in the GM of breastfeeding infants (P-value $=0.004,0.028$ in VD_B and CS_B) (Supplementary File 3). The CS_F group contained the most abundant unclassified taxon and the lowest Bifidobacterium load in the GM, while VD_B infants had the opposite trend. When receiving breastfeeding, the GM similarity between CS and VD infants was higher (from 0.18 to 0.52 ) than that with infants who experienced formula feeding (Fig. 1c).

\section{Discussion}

The assemblage of the GM during infancy is derived from the mother's faecal, vaginal and skin microbiota [19]. GM structures change dynamically over time in early life [2022]. Facultative anaerobic bacteria, such as Escherichia and Streptococcus, colonize the infant intestinal tract, consuming oxygen in the first few days after delivery, and then strict anaerobes, especially Bifidobacterium, thrive in the GM [3]. In this study, we identified 3 GM profiles that were dominated by an unclassified Escherichia/Shigella taxon, an unclassified Bifidobacterium taxon or Bifidobacterium. The
GM pattern gradually changed from Class 1 to Class 3, which is consistent with prior reports [21].

Maternal milk contains abundant nutrients, such as prebiotics, as well as beneficial bacteria, such as Bifidobacterium $[6,23]$. The key role of human milk in GM maturation has been previously emphasized [6, 7]. The enriched Bifidobacterium sp. could degrade human milk oligosaccharides (HMOs) [5, 24] to produce lactate and acetate, which maintain a low $\mathrm{pH}$ for digestive enzyme activation and serve as energy sources for colonocytes [25]. Human milk also facilitates later colonization of anaerobic microbial commensals, educating the host immune system and providing colonization resistance for opportunistic pathogens [24].

The positive contribution of human milk to GM development $[5,6]$ may partly explain why breastfeeding could restore the delayed GM development in CS infants towards that in VD as well as lower the risk of RTI and diarrhoea [26] Consistent with prior findings that GM development is successive, $[20,27]$ our study also identified no specific time point for breastfeeding-associated GM restoration.

Despite the additional insight into the GM restoration caused by breastfeeding, several limitations of our study should be noted. A small sample size may cause some bias in the analysis, and we are conducting a multicentre longitudinal study to confirm our preliminary findings. In an on-going project, we also enrolled infants who were fed formula with probiotics to confirm whether additive probiotics could better improve GM maturation and lower the risk of diseases.

Table 1 Characters' distribution of 41 enrolled infants

\begin{tabular}{|c|c|c|c|}
\hline & Breast feed & Formula feed & $p$-value \\
\hline \multicolumn{4}{|l|}{ Delivery Mode } \\
\hline Caesarean-section & 7 & 10 & \multirow[t]{2}{*}{0.279} \\
\hline Vaginal delivery & 14 & 10 & \\
\hline \multicolumn{4}{|l|}{ Gender } \\
\hline Female & 7 & 12 & \multirow[t]{2}{*}{0.087} \\
\hline Male & 14 & 8 & \\
\hline Gestational age (week) ${ }^{a}$ & $39.18 \pm 1.03$ & $39.36 \pm 1.19$ & 0.615 \\
\hline RTI-frequency in the first year ${ }^{a}$ & $2.57 \pm 1.03$ & $4.05 \pm 1.06$ & $<0.001$ \\
\hline Diarrhea-frequency in the first year ${ }^{a}$ & $1.14 \pm 0.79$ & $2.35 \pm 1.09$ & $<0.001$ \\
\hline
\end{tabular}

Represented by mean \pm SD 
This study revealed that breastfeeding could restore the delayed GM development of caesarean infants. The results expand the understanding of dynamic changes in the GM that occur in early life and provide new evidence to support the breastfeeding policy.

\section{Supplementary Information}

The online version contains supplementary material available at https:/doi. org/10.1186/s12887-020-02433-x.

\section{Additional file 1. \\ Additional file 2 \\ Additional file 3 .}

\section{Abbreviations}

CS: Caesarean-section; GM: Gut microbiota; OTUs: Operational taxonomic units; PERMANOVA: Permutational multivariate analysis of variance; RTI: Respiratory tract infection; VD: Vaginal delivery

\section{Acknowledgments}

We are grateful to the nursing staffs who contributed to sample collection. We also thank Nature English Editing Service for polishing the manuscript.

\section{Authors' contributions}

$\mathrm{LZ}$ and WD designed and managed the project. CG and $M L$ enrolled the children and performed the specimens sampling. LX, YZ and YW conduct DNA library extraction and construction. QZ and LZ performed the bioinformatics analysis, graph. CG and QZ interpreted the analysis results and wrote the manuscript. SL and DL guided the statistical analysis. All authors read and approved the final manuscript.

\section{Funding}

This work was supported by The Support Program of Science and Technology in Hebei province, China (No. H2018206310) and Shenzhen Science and Technology Project (No. JCYJ20170816170527583). The funding bodies played no role in the design of the study and collection, analysis, and interpretation of data and in writing the manuscript.

\section{Availability of data and materials}

The datasets generated and analysed during the current study are available in the GenBank database under accession number PRJNA576564, http:// www.ncbi.nlm.nih.gov/genbank/

\section{Ethics approval and consent to participate}

This study was approved by the Ethics Committee of the Third Hospoital of Hebei Medical University under approval number: 2010-009-1. All procedures performed in this study was in accordance with the ethical standards of the institutional and/or national research committee, as well as the 1964 Helsinki declaration and its subsequent amendments or comparable ethical standard. The guardians of enrolled infants gave written informed consents to participate this research project.

\section{Consent for publication}

Not applicable.

\section{Competing interests}

We declare no financial interests or conflicts.

\section{Author details}

'Department of Pediatrics, The Third Hospital of Hebei Medical University, No.139 Ziqiang Road, Shijiazhuang 050051, China. ${ }^{2}$ Department of Computer Science, City University of Hong Kong, Hong Kong 999077, China. ${ }^{3}$ School of Public Health, Peking University, No.38 Xueyuan Road, Beijing 100191, China. ${ }^{4}$ Department of Microbial Research, WeHealthGene Institute, Shenzhen 518000, China. ${ }^{5}$ Department of Information, The 960 Hospital of the Joint Logistic Support Force of the Chinese People's Liberation Army, Jinan 250031, China
Received: 28 May 2020 Accepted: 17 November 2020

Published online: 25 November 2020

\section{References}

1. Stewart CJ, Embleton ND, Marrs EC, et al. Temporal bacterial and metabolic development of the preterm gut reveals specific signatures in health and disease. Microbiome. 2016;4(1):67.

2. Tamburini S, Shen N, Wu HC, Clemente JC. The microbiome in early life: implications for health outcomes. Nat Med. 2016;22(7):713-22.

3. Mueller NT, Bakacs E, Combellick J, Grigoryan Z, Dominguez-Bello MG. The infant microbiome development: mom matters. Trends Mol Med. 2015;21(2): 109-17.

4. Praveen $\mathrm{P}$, Jordan F, Priami C, Morine MJ. The role of breast-feeding in infant immune system: a systems perspective on the intestinal microbiome. Microbiome. 2015:3:41.

5. Smilowitz JT, Lebrilla CB, Mills DA, German JB, Freeman SL. Breast milk oligosaccharides: structure-function relationships in the neonate. Annu Rev Nutr. 2014:34:143-69.

6. Pannaraj PS, Li F, Cerini C, et al. Association between breast Milk bacterial communities and establishment and development of the infant gut microbiome. JAMA Pediatr. 2017;171(7):647-54.

7. Fooladi AA, Khani S, Hosseini HM, et al. Impact of altered early infant gut microbiota following breastfeeding and delivery mode on allergic diseases. Inflammation Allergy Drug Targets. 2013;12(6):410-8.

8. Milani C, Duranti S, Bottacini F, et al. The First Microbial Colonizers of the Human Gut: Composition, Activities, and Health Implications of the Infant Gut Microbiota. Microbiol Mol Biol Rev: MMBR. 2017;81(4):e00036.

9. Backhed F, Roswall J, Peng Y, et al. Dynamics and stabilization of the human gut microbiome during the first year of life. Cell Host Microbe. 2015; 17(5):690-703.

10. Stewart CJ, Ajami NJ, O'Brien JL, et al. Temporal development of the gut microbiome in early childhood from the TEDDY study. Nature. 2018; 562(7728):583-8.

11. Yassour $M$, Vatanen $T$, Siljander $H$, et al. Natural history of the infant gut microbiome and impact of antibiotic treatment on bacterial strain diversity and stability. Sci Transl Med. 2016;8(343):343ra381.

12. Dominguez-Bello MG, Costello EK, Contreras M, et al. Delivery mode shapes the acquisition and structure of the initial microbiota across multiple body habitats in newborns. Proc Natl Acad Sci U S A. 2010;107(26):11971-5.

13. Wopereis H, Oozeer R, Knipping K, Belzer C, Knol J. The first thousand days intestinal microbiology of early life: establishing a symbiosis. Pediatr Allergy Immunol. 2014;25(5):428-38.

14. Martin $\mathrm{R}$, Makino $\mathrm{H}$, Cetinyurek Yavuz $\mathrm{A}$, et al. Early-life events, including mode of delivery and type of feeding, siblings and gender, shape the developing gut microbiota. PLoS One. 2016;11(6):e0158498.

15. Schloss PD, Westcott SL, Ryabin T, et al. Introducing mothur: open-source, platform-independent, community-supported software for describing and comparing microbial communities. Appl Environ Microbiol. 2009;75(23): 7537-41.

16. Guo C, Li Y, Wang $P$, et al. Alterations of gut microbiota in Cholestatic infants and their correlation with hepatic function. Front Microbiol. 2018;9: 2682

17. Edgar RC. Accuracy of microbial community diversity estimated by closed and open-reference OTUs. PeerJ. 2017:5:e3889.

18. Arumugam $M$, Raes J, Pelletier $E$, et al. Enterotypes of the human gut microbiome. Nature. 2011:473(7346):174-80.

19. Palmer C, Bik EM, DiGiulio DB, Relman DA, Brown PO. Development of the human infant intestinal microbiota. PLoS Biol. 2007:5(7):e177.

20. Backhed F, Roswall J, Peng Y, et al. Dynamics and stabilization of the human gut microbiome during the first year of life. Cell Host Microbe. 2015; 17(6):852.

21. Dogra S, Sakwinska O, Soh SE, et al. Dynamics of infant gut microbiota are influenced by delivery mode and gestational duration and are associated with subsequent adiposity. mBio. 2015;6(1):e02419.

22. Yang R, Gao R, Cui $S$, et al. Dynamic signatures of gut microbiota and influences of delivery and feeding modes during the first 6 months of life. Physiol Genomics. 2019:51(8):368-78.

23. Soto $A$, Martin $V$, Jimenez $E$, et al. Lactobacilli and bifidobacteria in human breast milk: influence of antibiotherapy and other host and clinical factors. $J$ Pediatr Gastroenterol Nutr. 2014;59(1):78-88. 
24. Lewis ZT, Totten SM, Smilowitz JT, et al. Maternal fucosyltransferase 2 status affects the gut bifidobacterial communities of breastfed infants. Microbiome. 2015;3:13.

25. Fukuda S, Toh $\mathrm{H}$, Hase $\mathrm{K}$, et al. Bifidobacteria can protect from enteropathogenic infection through production of acetate. Nature. 2011; 469(7331):543-7.

26. Bager P, Simonsen J, Ethelberg S, Frisch M. Cesarean delivery and risk of intestinal bacterial infection. J Infect Dis. 2010;201(6):898-902.

27. Ferretti $P$, Pasolli E, Tett $A$, et al. Mother-to-infant microbial transmission from different body sites shapes the developing infant gut microbiome. Cell Host Microbe. 2018;24(1):133-45 e135.

\section{Publisher's Note}

Springer Nature remains neutral with regard to jurisdictional claims in published maps and institutional affiliations.

Ready to submit your research? Choose BMC and benefit from:

- fast, convenient online submission

- thorough peer review by experienced researchers in your field

- rapid publication on acceptance

- support for research data, including large and complex data types

- gold Open Access which fosters wider collaboration and increased citations

- maximum visibility for your research: over $100 \mathrm{M}$ website views per year

At BMC, research is always in progress.

Learn more biomedcentral.com/submissions 